\title{
Trigger Factors, Allergens and Allergy Testing in Atopic Dermatitis
}

\author{
Evmorfia Ladoyanni \\ Department of Dermatology, Dudley Group Hospitals, Corbett Hospital \\ Vicarage Road, Stourbridge, West Midlands \\ United Kingdom
}

\section{Introduction}

Atopic dermatitis (AD) is a chronic inflammatory skin disorder affecting between $10-20 \%$ of children and $1-3 \%$ of adults in the general population. It is associated with high morbidity and has major public health implications. AD is associated with hyper-reactivity of the skin to environmental trigger factors that are harmless in normal individuals. Major contributors to this hyperactivity are the many immune and inflammatory changes taking place in the skin and peripheral blood in AD individuals. AD can be classified into several subgroups, each with different immune and pathological features, suggesting a multifactorial disease and heterogeneity. Historically two types of $\mathrm{AD}$ have been identified. They include the "extrinsic or allergic" type of $\mathrm{AD}$, which is characterized by Immunoglobulin $\mathrm{E}$ (IgE) mediated sensitization (immediate, type I hypersensitivity) and occurs in $70-80 \%$ of individuals. The "non-allergic or intrinsic" type of AD affects $20-30 \%$ of individuals and is defined by a more T-cell driven feature (delayed, type IV hypersensitivity), with low IgE levels and absent IgE sensitization. More recently a transition from the intrinsic to the extrinsic type has been reported in young children, and AD individuals can show a combination of above mentioned phenotypes or complete absence of the known features. The clinical diagnosis of $\mathrm{AD}$ does not cause major difficulties as there are fairly well defined criteria. Identifying the underlying pathophysiology for the affected individual can be more of a challenge, as there is no gold standard test available for AD and the AD individual might move from one pathophysiological "type" of AD to another. Clinically AD is divided into acute, subacute and chronic forms with correlated histopathological changes. The transition from one clinical form to another is fluent and can be very rapid. AD individuals frequently give a personal and or a family history of asthma and allergic rhinitis, the so called atopic triad. Exacerbation of one of the disorders in this triad can cause concomitant exacerbation of the other two suggesting some underlying common pathways and interdependence (Reitamo et al., 2008).

Predisposing factors for AD are multifactorial and involve genetic susceptibility, defects in skin barrier function, immune dysregulation, western life style and exposure to environmental trigger factors. The development of disease is an interplay of gene-gene and gene-environment interactions. The early age of onset and familial predisposition as well as the high concordance rate of $\mathrm{AD}$ in monozygotic (77\%) and dizygotic (15\%) twins suggest a 
genetically determined primary defect probably affecting the skin barrier thus playing a key role in the development of AD (Burns et al., 2004). The integrity of the skin barrier involves several components, including regulation of proteolysis of corneodesmosomes, the lipid lamellae and generation of natural moisturizing factor (MNF) from the breakdown products of the structural protein filaggrin. An imbalance of these components makes the epidermal barrier more susceptible to irritants, which in turn can lead to a disturbance of skin barrier function allowing penetration of microbes and allergens into the epidermis and dermis interdependence (Reitamo et al., 2008). In a recent meta-analysis filaggrin gene defects were shown to increase the risk of developing allergic sensitization, $\mathrm{AD}$, and allergic rhinitis. The presence of filaggrin gene mutations correlated strongly with disease severity and treatment failure, and also increased the risk of asthma in AD patients (Van den Oord \& Sheikh, 2009). IgE is composed of two identical heavy and light chains, that form the constant Fc domain and the antigen-binding sites, through which the $\operatorname{IgE}$ molecule binds to its cell surface receptors. Raised serum IgE levels are most commonly seen in parasitic infections as a defensive response. IgE mediated hypersensitivity is largely regulated by $\mathrm{T}$ lymphocytes. AD is generally thought to be due to an imbalance between Th1 and Th2 cells, with a Th2 cell predominant immune profile in its active stage. Th2 cells produce interleukin (IL) -4, IL5, IL-6 and IL-13, which induce IgE production and activation of eosinophils, producing the acute signs and symptoms of AD.

In general various cells contribute to the underlying pathomechanism of AD including Langerhans cells (LC), macrophages, T cells, B cells, keratinocytes, endothelial cells, eosinophils and mast cells. These cells communicate with each other in Th2 predominant cytokines as well as chemokines, prostanoids, proteases and reactive oxygen species products. High affinity IgE receptors play a crucial role in promoting this process.

A number of environmental factors can trigger and perpetuate the inflammatory skin cascade in AD including irritants, foods, aeroallergens, infection. All four classic Coombs' classification types of hypersensitivity (type I-IV) have been implicated in the pathophysiology of AD, including pseudo allergy. The hypersensitivity types can occur either alone or in combination in the AD individual. In the extrinsic type of AD high levels of specific IgE antibodies and raised total IgE levels in peripheral blood have been significantly associated with severity of dermatitis (Reitamo et al., 2008 \& Burns et al., 2004).

\section{Trigger factors, allergens and allergy testing in atopic dermatitis}

The rate of increase in prevalence in AD in recent years is too rapid to be accounted for by changes in population genetics. Therefore environmental factors are the most likely modulating influences. The two principal aspects that have attracted attention are pollution and microbes. The identification of relevant pollutants that might contribute to the expression of atopic phenotype is still confused. Interaction with environmental microbes may be important in the causation of $\mathrm{AD}$ in a number of ways. Early-life exposure may condition maturation of the immune system so that apparent dysregulation associated with IgE production and allergy formation does not occur. Significant differences have been observed in the prevalence of allergies between urban and rural population within one country and observations confirm greater allergy sensitization in first-born and a lower frequency in children from large families. Evidence suggests that microbes entering via fecal-oral route have a greater protective effect against the development of atopic disease than those entering via the respiratory route (Burns et al., 2004). 
AD has a Th2 cell predominant immune profile in its active stage. Th2 cells produce IL-4, IL-5, IL-6, IL-13, which induce IgE production and activation of eosinophils, producing the acute signs and symptoms of AD. Genetic factors, reduced bacterial stimulation in early infancy and a disruption in skin barrier and function are thought to contribute to the Th1-Th2 cell imbalance of skin in AD. Eczematous skin lesions evolve as a result of complex interactions between $\operatorname{IgE}$ bearing antigen presenting cells, $\mathrm{T}$ cell activation, mast cell degranulation, keratinocytes, eosinophils, and a combination of immediate and cellular immune response. A number of environmental factors have been reported to induce and perpetuate this inflammatory response of the skin including food and aeroallergens, microbes and irritants. All four classic types of hypersensitivity reactions have been implicated in the pathophysiology of AD including pseudo allergy. They can occur either alone or in combination in AD individuals (Reitamo et al., 2008 \& Burns et al., 2004).

$\mathrm{AD}$ is a common chronic skin condition associated with high morbidity and major public health implications. As prevention of disease is not yet a real option, reducing morbidity is main aim of treatment. Identifying the underlying pathophysiology of the individual's AD is very crucial. To date no standard test is available to diagnose AD. Sensitization to various allergens is a major part of triggering and perpetuating the inflammatory skin response in AD. Various tests have been developed to investigate the underlying type(s) of hypersensitivity reaction(s) involved in AD patients. None of the available tests so far have proven sensitive and specific enough to identify reliably relevance between clinical disease and test result. Precise understanding of these tests including their limitations together with accurate correlation of patient symptoms and signs are required in order to differentiate between allergy, intolerances and hypersensitivities, and achieve an appropriate clinical diagnosis (Robinson \& Smart, 2008).

Allergy tests commonly used in practice include measurement of total levels of $\operatorname{IgE}$ and allergen specific IgE levels in serum (radio-allergosorbent test/RAST), skin prick testing (SPT) and atopy patch testing (APT). A different positive predictive cut off point exists for each allergen tested respectively and similar test results do not imply a similar clinical reaction to each allergen (Robinson \& Smart, 2008).

SPT is performed to detect the presence of allergen specific IgE to foods, aeroallergens, antibiotics and latex. A drop of a solution containing the allergen is applied to the skin and a lancet used to prick the skin. The allergen binds to IgE on mast cells causing degranulation and the release of histamine. This manifests as a weal and flare reaction, the diameter of which can be measured. SPT is easy to perform and results are immediately available. It can trigger off allergic reactions including anaphylaxis. Various medications including antihistamines, H2-antagonists, tricyclic antidepressants and neuroleptics can interfere with SPT and need to be withheld prior to testing. A positive SPT result indicates only sensitization and does not always equate to clinically relevant allergy. It must be interpreted in the context of clinical history, clinical signs and allergen exposure. The size of the weal does not necessarily correlate with severity of clinical reaction (Robinson \& Smart, 2008 \& Goodwin, 2008). The RAST detects free allergen specific IgE in serum. It is less sensitive and specific than SPT and is particularly useful where SPT is contraindicated. Testing is available for a wide range of food and environmental allergens. The RAST results are not affected by prior drug use and can be performed in patients with widespread skin disease. The RAST can be reported semi quantitatively as a score or as quantitative measurement by using the CAP RAST technology. The process involves an allergy-impregnated disc being incubated with patient's serum. Allergen specific IgE, if present binds to allergen. The disc is 
then incubated with radio-labeled anti $\operatorname{IgE}$ and radioactivity measured to give level of specific IgE present to particular allergen. Positive RAST results indicate the presence of IgE to an allergen or cross reacting allergen, and are difficult to interpret in the presence of high levels of total $\operatorname{IgE}(>1000 \mathrm{kU} / \mathrm{L})$. Results therefore must always be interpreted in the context of clinical history (Robinson \& Smart, 2008 \& Goodwin, 2008).

APT is epicutaneous application of inhalant and food allergens on unaffected skin of AD individuals eliciting a delayed, type IV hypersensitivity reaction. It was traditionally developed to investigate association between AD and allergy to aeroallergens and proved in that context to have a higher specificity when compared to SPT and RAST. More recently the APT has been used to supplement the SPT in the diagnosis of food allergy, in an attempt to identify delayed reactions to food products. Reproducibility of APT results raises unsolved issues and is to date poor for most food allergens and good for inhalant allergens. Variables that can strongly influence results are allergen concentration, skin site and devices used for allergen application as well as reading time and/or criteria for defining positive reactions. These variables seem to be specific for each allergen (Lipozencić \& Wolf, 2010).

- Allergen concentration not standardized

- Different vehicles used

- Differences in skin sites and pre-treatment of skin

- Differences in reading time and duration of patch testing

Table 1. Factors affecting reproducibility of APT

Patch testing (PT) is an epicutaneous and the gold standard test in the diagnosis of contact allergic dermatitis (CAD). It is not a routine investigation for the diagnosis of AD, but ideal to rule out contact allergy (e.g. to topical medication) in the presence of AD. Recent evidence suggests that $\mathrm{CAD}$ is as common in $\mathrm{AD}$ individuals as in the general population and PT is recommended in $\mathrm{AD}$ patients with recalcitrant disease, hand eczema and in children (Fonacier \& Aquino, 2010).

Oral food challenges are the "gold standard" for the diagnosis of food allergy. They can be open, single or double blinded, or placebo controlled. A range of vehicles can be used to disguise food (liquids or solid). Tolerance of a serving size of a food is generally considered evidence of lack of reactivity. In AD oral food challenges should be performed in a doubleblind placebo-controlled manner (Robinson \& Smart, 2008).

Negative RAST, SPT and APT results have a high negative predictive value and are fairly reliable methods of excluding allergy. Positive results however require careful consideration and correlation with clinical history and clinical presentation (Robinson \& Smart, 2008 \& Goodwin, 2008).

\subsection{Aeroallergens}

Clinical manifestations of allergy to aeroallergens involve allergic rhinitis, asthma and exacerbation of AD. Patients with atopic predisposition can express one or all of these clinical manifestations. Filaggrin gene defects have been shown to increase the risk of developing allergic sensitization, AD, and allergic rhinitis (Van den Oord \& Sheikh, 2009). A variety of inhalants have been implicated in exacerbations of AD including house dust mite 
(HDM), dust mites, HDM feces, animal dander (cat and dog), molds and pollens. Sensitization to aeroallergens appears to be more prevalent in older children and adults, leading to aggravation of AD after bronchial inhalation. Allergen specific T- cells have been identified in affected skin of AD to grass pollen, birch pollen and HDM supporting the concept of a T-cell mediated specific immune response to inhalant allergens with clinical implications. Sensitization to inhalant allergens is thought to occur via skin as well as respiratory route. Allergens penetrate the disturbed epidermal barrier fix on the specific IgE present on LCs and activate them. The activated LCs migrate to a lymph node and present the allergen to $\mathrm{T}$ cells, provoking an eczematous reaction. The appearance of eczematous skin lesions is due to cytotoxic T-cell activity and not to the aeroallergen specific IgE. Repeated epicutaneous allergen application has been shown to elicit eczematous lesions in AD individuals (Reitamo et al., 2008 \& Goodwin, 2008).

\section{Transcutaneous route}

Mite allergen activates LCs via IgE fixation

Documented presence of HDM on skin

Induction of eczematous lesions following APT

\section{Respiratory route}

Flare up of AD following allergen inhalation

Induction of new eczematous skin lesions upon allergen inhalation

Table 2. Routes of sensitization aeroallergens in AD

The respiratory route in the induction of new skin lesions may be relevant in a subset of patients with concomitant allergic asthma. Early studies reported that inhalation of house dust or pollen extract can provoke exacerbations of AD. Skin lesions have also been shown to develop in AD individuals after placebo-controlled bronchial provocation of HDM, cat allergen or tree pollen. Aggravation of eczema is more pronounced in AD individuals with concomitant allergic asthma than in patients suffering from $\mathrm{AD}$ alone. Two possible explanations have been offered for this phenomenon. Allergen exposed in airways enters the circulation and is transported to skin activating there the inflammatory cascade. Another possibility is allergen induced airway inflammation causes release of mediators from inflammatory cells in skin already primed for AD. Substantial clinical improvement has been reported to occur when sensitized AD individuals are exposed to environments lacking the allergens to which they react (Reitamo et al., 2008\& Burns et al., 2004).

Mites belong to the most potent allergen sources and are considered a perennial allergen. Thirteen mite species have been identified in house dust so far, the two commonest mites in homes worldwide include Dermatophagoides pteronyssimus and Dermatophagoides farinae. Beds and overstuffed furniture are main foci for breeding dust mites. Airborne level of mite allergen during sleep in bedroom is tenfold higher than that found in living rooms of same house during daily activities. Mite allergens persist for months after death of mite. Exposure to high doses of mite allergen in early infancy has been associated with higher incidence of developing eczema. Efforts to create dust mite free environment yielded significant 
improvement in clinical symptoms although not complete remission. AD patients not sensitized or not exposed to mites benefited as much from exposure to mite free environment as those with mite sensitization and/or exposure.

- Evidence of aeroallergen specific T cells invading the skin

- Presence of aeroallergen specific $\operatorname{IgE}$

- Exacerbation of AD following exposure to aeroallergen

- Exacerbation of AD following epicutaneous aeroallergen application

- New skin lesions induced by APT

- Clinical improvement in aeroallergen poor environment

Table 3. Factors implicating aeroallergens underlying pathomechanism of AD

Many AD patients are highly sensitized to aeroallergens and experience precipitation of AD upon epicutaneous or bronchial allergen exposure. RAST and SPT help to verify or exclude the presence of $\operatorname{IgE}$ mediated hypersensitivity, by measurement of allergen specific $\operatorname{IgE}$ in serum and evaluation of IgE bound on mast cells. Neither test procedure considers those AD patients characterized by cell-mediated sensitization. The APT was established as a provocation test for a subgroup of $\mathrm{AD}$ patients to study the induction of eczema by aeroallergens after 24-72 hours. It is similar to nasal or bronchial provocation test in allergic rhinitis and asthma. Allergen specific IgE is not obligatory for a positive APT reaction. APT has shown a higher specificity towards inhalants (69-92\%) when compared to SPT (44-53\%) and serum specific IgE (42-64\%), suggesting a more appropriate screening role for SPT and RAST in this context. APT does not replace the classical methods of diagnosis of IgEmediated allergy. APT are usually negative in individuals with respiratory allergy and healthy subjects pointing to AD specific triggering mechanisms required to produce APT positivity. Much effort has been undertaken to standardize APT procedures. The protocol of the European task Force on Atopic Dermatitis (ETFAD) provides a standardized APT technique (Ronchetti et al., 2008).

Despite above impressive data on APT as a diagnostic tool, final scientific proof for the relevance of aeroallergens as identified by positive APT, for clinical manifestation of AD is still missing. The question on how well APT can identify AD patients who would benefit from allergen avoidance remains to be answered.

- Suspicion of aeroallergen symptoms without proof of positive specific IgE or positive SPT

- $\quad$ Severe and/or persistent AD with unknown trigger factors

- Multiple IgE sensitizations without proven clinical relevance in patients with eczema

Table 4. ETFAD consensus on APT indications

In summary sensitization to inhalant allergens is a major contributing factor to the pathogenesis and perpetuation of $\mathrm{AD}$. It is found more commonly in older children and later life of $\mathrm{AD}$ individuals and is demonstrated by presence of aeroallergen specific $\mathrm{IgE}$ and positive SPT to aeroallergens. Bronchial inhalation challenge, RAST, SPT, and APT to 
aeroallergens, are the main tests available for investigation. Using these tests in combination and in consideration of clinical history and signs is likely to yield a higher clinically relevant result. Negative results of above investigations are fairly reliable methods of excluding allergy.

\subsection{Food allergens}

The prevalence of $\mathrm{AD}$ and food allergies (FA) appears to have increased in recent years in many western countries. In this context it is important to define the terms. FA and food hypersensitivity $(\mathrm{FH})$ indicate an adverse clinical reaction to food due to interaction of food proteins with one or more immune mechanisms. Food intolerance (FI) is the result of nonimmunological reactions to foods and food additives (Reitamo et al., 2008).

- Food allergy due to IgE mediated mechanism (Coombs' classification, type I)

- Food allergy involving other immunological mechanisms ( type IV)

- Non-allergic food intolerance (pharmacological, metabolic, or toxic reaction to food)

- Food aversion (often non-specific symptoms, unconfirmed by blinded food challenge)

Table 5. Types of adverse reactions to foods

FA has the greatest incidence in infancy and early childhood. Around $8 \%$ of children are thought to develop adverse reactions to food, most of them within the first year of life. The prevalence of FA varies between regions and is influenced by culture and genetic factors. The spectrum of food allergens has remained relatively unchanged despite the rise of FA incidence. Seven food items account for $90 \%$ of FA in children. These include cow's milk, egg, peanuts, soybeans, wheat, fish, and tree nuts. By three years of age the majority of children will have developed tolerance to these food items with the exception of peanuts/ tree nuts and seafood. In adults, IgE mediated FA is rare. The main food allergens in adults are peanuts, tree nuts, fish and shellfish (Kim, 2008).

\begin{tabular}{|c|c|}
\hline Infants and children & Adults \\
\hline Cow's milk & Peanut \\
Egg & Tree nuts \\
Peanut & Fish \\
Tree nuts & Shellfish \\
Soy & \\
Wheat & \\
Fish & \\
\hline
\end{tabular}

Table 6. Major food allergens in children and adults

In general high-protein food allergens are considered to be more allergenic. Most food allergens are water-soluble glycoproteins that are particularly resistant to food processing, cooking and digestion. 


\begin{tabular}{|c|c|}
\hline Food Item & Protein \\
\hline Cow's milk & $\begin{array}{c}\text { Caseins } \\
\mathrm{a}_{1} \text {-casein } \\
\mathrm{a}_{2} \text {-casein } \\
\beta \text {-casein } \\
\text { K-casein } \\
\gamma \text {-casein } \\
\text { Whey proteins } \\
\beta \text {-lactoglobulin } \\
\text { a-lactalbumin } \\
\text { Bovine serum } \\
\text { albumin (BSA) }\end{array}$ \\
\hline Egg white & $\begin{array}{c}\text { Ovomucoid } \\
\text { Ovalbumin } \\
\text { Ovotransferrin/conalbumin } \\
\text { Lysozyme }\end{array}$ \\
\hline Peanut & $\begin{array}{l}\text { Vicillin } \\
\text { Conglutin } \\
\text { Glycinin }\end{array}$ \\
\hline Soy bean & $\begin{array}{c}\text { Gly } \mathrm{m} 1 \\
\text { Trypsin inhibitor }\end{array}$ \\
\hline
\end{tabular}

Table 7. Common food allergens

FA in infancy is thought to be due to a failure of the gastrointestinal tract (GIT) to develop oral tolerance (OT). No down regulation occurs of GIT immune system in its responsiveness towards ingested soluble food antigens. Factors predisposing to impaired OT include increased antigen uptake, decreased production of secretory $\operatorname{IgA}$, and an imbalance towards Th2 response. Other factors include dose and frequency of antigen exposure, biological characteristics of antigens as well as early intestinal inflammation affecting gut permeability and mucosal antigen uptake. IgE sensitization can occur as early as during fetal period and maternally ingested antigens can be excreted in the breast milk. An additional mode of sensitization to food allergens in AD individuals may involve the impaired skin barrier function, so that sensitization to food allergens may occur even though the food in question has not yet been integrated into the diet (Reitamo et al., 2008 \& Kim, 2008).

Immunologically triggered FA can clinically present as immediate (oral allergy syndrome, urticaria, angioedema, bronchospasm and anaphylaxis), intermediate (predominantly GIT symptoms), and late-onset reactions (AD flares, development of cough and wheeze). 
- Specific food antigen can be identified

- Timing of symptoms closely related to food intake

- Symptoms can involve more than one organ (oral itching and/or swelling, nausea, vomiting, abdominal pain, diarrhea, asthma, rhinitis, urticaria, angioedema, anaphylaxis)

- Positive personal or family history of other atopic disorders

Table 8. Factors suggesting classic IgE mediated food allergy

FA is the earliest manifestation of atopy affecting both breast- and formula-fed infants. Early sensitization to food allergens is typically transient. It affects AD individuals mainly in the first 2 to 3 years but particularly in the first 12 months of life and is often followed by inhalant sensitization as demonstrated by presence of aeroallergen specific $\operatorname{IgE}$ and positive SPT to aeroallergens. These may in turn contribute to ongoing eczema later in life (older children and adults). Multiple studies have established that approximately $35 \%$ of children with moderate to severe $\mathrm{AD}$ have FA. Double-blind placebo-controlled food challenge (DBPCFC) is the gold standard for diagnosis of FA. Children with AD, that were assessed for FA by DBPCFC showed reactions in $40 \%$ of challenges. Of the positive challenges skin symptoms occurred in $84 \%$ of $\mathrm{AD}$ subjects, $56 \%$ showed either skin, GIT or respiratory symptoms and only $30 \%$ developed isolated skin reactions. Children with positive DBPCFC were younger than those without. Generally, the younger the patient and the more severe the AD, the more likely FA is to be a causative factor (Reitamo et al., 2008 \& Kim, 2008). In adults with AD IgE-mediated FA plays only a minor role (1-3\%). In this group birch pollen related food allergens have been shown to be a major predisposing factor. In recent years pseudo allergens have been reported to exacerbate symptoms in adults with $\mathrm{AD}$, although it is still controversial if these observations always reflect a true pseudo allergic underlying pathomechanism. Pseudo allergic reactions are more known in the clinical context of urticaria and angioedema. To date no reliable laboratory or skin tests are available to diagnose pseudo allergy, so that for the identification of possible pseudo allergen trigger factors elimination diet and oral challenge are the norm (Reese et al., 2009).

Approximately $85 \%$ of AD individuals have specific IgE to food- and aeroallergens, making diagnosis of FA based on laboratory testing alone inadvisable. The importance of a careful history cannot be overemphasized. The history combined with diagnostic tests, should point toward a few possible offending foods or groups of food products. Food allergen specific IgE is a useful tool in the assessment of FA. A different positive predictive cut off point exists for each allergen tested and similar test results do not imply a similar clinical reaction to different allergen. Higher levels of specific IgE indicate increased likelihood of allergic reaction with exposure. Levels though cannot predict the severity of allergic reaction. In general positive RAST results only indicate the presence of IgE to an allergen or crossreacting allergen and may not correlate with true clinical reactions. Patients with AD can have very high levels of non-specific IgE, which can be detected and give rise to false positive results (Robinson \& Smart, 2008 \& Goodwin, 2008 \& Kim, 2008).

SPT in the diagnosis of FA in infancy is thought to be unreliable. It can provide rapid results with high sensitivity, and has a high negative predictive value $(>95 \%)$, with a negative skin test essentially excluding IgE- mediated FA. In contrast the positive predictive value is 
$<50 \%$; suggesting that an isolated positive SPT cannot be predictive for FA. A cut off weal diameter of $6-8 \mathrm{~mm}$ has been demonstrated to be associated with a positive oral food challenge (predictive value $>95 \%$ ). In this context SPT can reduce the need for oral food challenges by $23 \%$ when compared to RAST testing. SPT with fresh fruits and vegetables is a better predictor of clinical relevant reaction in comparison to the use of commercial extracts for SPT (Robinson \& Smart, 2008 \& Goodwin, 2008 \& Kim, 2008).

FA in children with AD is thought to be an IgE- and T-cell mediated phenomenon, making the use of the APT a useful diagnostic tool. Clinical studies have demonstrated the APT to be particularly useful in predicting late-phase clinical reactions. However different studies have published contradicting results regarding sensitivity of unstandardized food APT and further clinical studies are required for standardization and patient group selection for food APT. APT cannot replace oral food challenges. Finally APT in conjunction with positive RAST and positive SPT to food allergens has been shown to reliably predict positive food challenges (Robinson \& Smart, 2008 \& Goodwin, 2008 \& Kim, 2008 \& Ronchetti et al., 2008). In summary, a proper dietary and clinical history combined with available testing are more likely to yield a clinical relevant result. Involvement of a dietitian is paramount in AD patients complaining of FA in order to avoid malnutrition in the long run.

\subsection{Microbial allergens}

Interaction with environmental microbes may be important in the causation of AD in a number of ways. Early-life exposure may condition maturation of the immune system so that apparent dysregulation associated with IgE production and allergy formation does not occur. The possible role of microbes in the early maturation of the immune system may be the major factor that could explain the differences between western and developing world regarding the incidence of atopy and allergic diseases. Microbes entering via fecal-oral route have been shown to have a greater protective effect against development of allergic diseases than those entering via respiratory route. Early exposure to Hepatitis A virus, Helicobacter pylori or Toxoplasma gondii has been associated with a reduced risk for atopy by $60 \%$. The burden of exposure of microbial endotoxins in early infancy is thought to play a major role in driving the immune system towards protective responses and away from nuisance responses that are associated with allergy (Burns et al., 2004). A number of observations suggest the relevance of skin flora in clinical manifestation of AD. In individuals with atopic phenotype, eczema may be induced or exacerbated by staphylococcal toxins or by the presence of Malassezia yeasts on the skin.

Staphylococcus aureus is found on the skin of $90 \%$ of chronic AD individuals, but only on $5 \%$ of healthy subjects. When present on healthy normal skin S. aureus is usually low in number and mainly confined to intertriginous areas and nasal nares. The presence of $S$. aureus on atopic skin depends on the skin lesion. S. aureus has been isolated in $55-75 \%$ of clinically unaffected AD skin, $85-91 \%$ of chronic lichenified lesions and $80-100 \%$ of acute exsudative eczematous lesions. The density of $S$. aureus on acutely inflamed AD skin can be 1000-fold higher than on uninvolved skin in AD individuals suggesting a good correlation between the degree of bacterial colonization and clinical disease severity

The ability of $S$. aureus to cause human disease is dependent on production of cell surface adhesions, antiphagocytic factors and secreted exotoxins, whose function appear to be both securing nutrient for microbes and delaying function of immune system. Among the factors secreted by $S$. aureus is the large family of superantigen exotoxins. 
The factors influencing colonization and infection of S. aureus in AD skin are not yet well understood and likely to be multifactorial. Defective skin barrier function, reduced content of skin lipids, loss of certain innate antibacterial activities via changes of antibacterial peptides on skin, and a more alkaline $\mathrm{pH}$ of skin surface are all thought to play a role in this context. Antimicrobial peptides such as $\beta$-defensins and cathelicidins are critical elements of the skin's innate immunity. These peptides eliminate not only $S$. aureus, but also fungal, viral and other bacterial pathogens. The decreased antimicrobial peptide levels in AD skin are not caused by intrinsic defect in keratinocyte production but rather to inhibition mediated by the Th2 cytokine milieu in the AD microenvironment (Schlievert et al., 2008).

Scratching disturbs the epidermal barrier and releases pro-inflammatory cytokines that up regulate the extracellular matrix molecules to act as adhesins to $S$. aureus. Cracks in the skin expose underlying extracellular matrix molecules to S. aureus attachment (Reitamo et al., 2008).

- Disturbed skin barrier and function

- $\quad$ Reduced content of skin lipids

- $\quad \mathrm{pH}$ of skin surface more alkaline

- Increased adherence of $S$. aureus to skin due to raised fibronectin and fibrinogen (so called adhesins)

- $\quad$ Reduced keratinocyte production of $\beta$-defensins and LL-37 (antimicrobial peptides)

Table 9. Factors influencing colonization and infection with S. aureus in AD

Staphylococcal super antigens include staphylococcal enterotoxins, classically the common cause of food poisoning and non menstrual toxic shock syndrome (TSS), and TSS toxin1 (TSST-1), the cause of both menstrual and non menstrual TSS. Staphylococcal enterotoxin serotypes A-E (SEA-SEE) and SEG-SEQ are well documented in the literature. Super antigens are defined by their ability to stimulate cytokine release from both $T$ cells and macrophages. They bind directly without antigen processing to constitutively expressed HLD-DR molecules on professional antigen-presenting cells precipitating marked $\mathrm{T}$ cell stimulation (Macias et al., 2011).

Most AD individuals show raised specific IgE levels in serum against staphylococcal superantigens and rarely make $\operatorname{IgE}$ against constituents of $S$. aureus cell wall. They also demonstrate super antigen specific $\operatorname{IgE}$ on their skin. Basophils from patients with superantigen specific IgE release histamine on exposure to relevant superantigen, but not when exposed to super antigens they are not sensitized to. A correlation between presence of superantigen specific IgE and clinical AD severity has been identified and colonization with superantigen producing S. aureus is greatest with IgE to staphylococcal superantigen. Skin homing peripheral $\mathrm{T}$ cells have also been shown to respond to superantigen and contribute to eosinophilia and IgE production in AD. Epicutaneous application of SEB to unaffected AD skin is followed by exacerbation of eczema suggesting that superantigens can precipitate and sustain the dermal inflammatory cascade in AD individuals. Combined treatment of AD with antibiotics and corticosteroids is more effective than corticosteroid treatment alone suggesting that $S$. aureus secrets products that can induce steroid resistance (Schlievert et al., 2008). In a recent study $S$. aureus strains isolated from steroid resistant AD showed an ability to 
produce larger numbers of superantigen types per organism. There was also dysregulated production of super antigens and production of unusual superantigen combinations. Reduction of $S$. aureus on AD skin controls the skin inflammation which predisposes to $S$. aureus colonization and/or infection. Discrepancies in antibiotic sensitivity pattern have been documented among $S$. aureus strains colonizing different sites of AD skin (affected and unaffected) and also in $\mathrm{AD}$ individuals with prior antibiotic therapy. Repeat microbial susceptibility testing on different body sites is therefore recommended (Kedzierska et al., 2008).

- Efficacy of topical antiseptic treatment

- Efficacy of topical antibiotic therapy

- Efficacy of application of gammaglobulins

- Efficacy of oral antibiotic and antifungal therapy

Table 10. Relevance of skin flora for AD

In recent years there is an increasing body of evidence suggesting that yeasts particularly of the genera of Malassezia and Candida can be relevant for AD pathogenesis. Many studies have demonstrated colonization of $\mathrm{AD}$ skin with Malassezia yeasts with subsequent deterioration of clinical disease (Darabi et al., 2009). Malassezia yeasts are members of the normal human skin flora, and often associated with different dermatological disease. The concentration of yeasts on skin does not have to be raised in AD individuals, suggesting that skin barrier dysfunction and typical changes in skin inflammatory response in AD play a major role. Great variation in density and presence of Malassezia yeasts in different sites of AD skin has been documented with the highest concentrations affecting the scalp and upper trunk and the lowest on hands. Sensitization to Malassezia yeasts have been exclusively demonstrated in AD individuals, via SPT with Malassezia extracts and the presence of Malassezia specific IgE antibodies. The density of the yeasts reduces with progressing age, although the yeast cells are larger in adults than in children. Malassezia specific IgE in serum has been identified in $20-100 \%$ of AD subjects depending on trial. Prevalence was highest in adults with head and neck AD (HNAD) and lowest in children with AD. A strong correlation between Malassezia specific IgE levels and severity of HNAD has been found, with Malassezia specific IgE being a good marker for HNAD (Darabi et al., 2009). Positive SPT to Malassezia extracts have been reported in $13.5-79 \%$ of AD individuals, compared to small minority in individuals with other Malassezia induced skin conditions. This suggests an exclusive presence of Malassezia sensitization in AD subjects. Malassezia antigens have been used to APT in some trials and positive results were observed in AD patients suggesting a possible role of Malassezia yeasts in eliciting and perpetuating eczema in already sensitized AD individuals (Reitamo et al., 2008 \& Darabi et al., 2009). Malassezia extracts are not yet part of the standardized APT panel provided by ETFAD. No consistent correlation has been shown between HNAD and positive APT. In many trials oral antifungal therapy has been shown to improve clinical manifestations of $\mathrm{AD}$, particularly in the context of HNAD, exacerbations during adolescence and young childhood, severe AD recalcitrant to conventional therapy, and presence of other atopic diseases. It is not clear yet if this is due to the anti-inflammatory effects of antifungals or due to their antifungal 
properties. Despite the available positive data on Malassezia species and AD there is not convincing proof for the importance of this yeast in AD pathogenesis. Malassezia sensitization has been reported in both types of $\mathrm{AD}$, extrinsic and intrinsic, and skin barrier dysfunction in $\mathrm{AD}$ enabling easier access and therefore cross reactivity between human and fungal manganese superoxide dismutase might be another explanation for the sensitization to Malassezia yeasts seen in AD.

Colonization with Candida albicans has been found in higher numbers on involved and uninvolved skin of AD individuals than on normal subjects. More importantly colonization of the gastrointestinal tract with Candida yeasts was identified in $70 \%$ of AD patients compared to $54 \%$ of healthy volunteers. $22-94 \%$ of AD individuals have shown positive SPT to $C$. albicans and positive correlation existed between the number of positive SPTs and clinical disease severity. C. albicans specific IgE has been reported in $25-88 \%$ of AD patients and cross reactivity to Malassezia has been described. Interestingly PT with C. albicans extracts have shown fewer positive results than the healthy controls. In summary the evidence on $C$. albicans is too small to allow any conclusions about its role in AD pathogenesis (Reitamo et al., 2008).

\subsection{Autoallergens}

IgE autoreactivity has been implicated in the immune pathogenesis and in particular perpetuation of $\mathrm{AD}$. Striking similarities between environmental allergens and human proteins have been identified on molecular analysis of allergens, and IgE-reactive autoantigens against human proteins have been cloned from human epithelial copy DNA expression libraries. Autoantigens have been detected in IgE-mediated immune complexes in serum of $\mathrm{AD}$ individuals and release of autoallergens following tissue (skin barrier) damage has been reported. More importantly reduced levels of $\operatorname{IgE}$ autoallergen levels have been noted following successful treatment of AD. It is possible that following initiation of the AD immune and inflammatory cascade in skin and peripheral blood by environmental allergens, these mechanisms are then partially perpetuated by autoallergens. More studies are required in the future to allow any sensible conclusion (Reitamo et al., 2008).

\subsection{Contact allergens}

It is clear that $\mathrm{AD}$ individuals are at greater risk in developing irritant contact dermatitis than non-atopic subjects, as contact allergens are likely to penetrate the defective skin barrier more easily. In this context hand eczema has been reported to occur in higher frequency in $\mathrm{AD}$ patients. AD individuals, who are particularly employed in occupations involving wetwork are prone to develop an intractable hand dermatitis in their occupational setting leading to considerable occupational disability. Urticaria is frequently encountered in AD individuals, and an allergic basis is found more often in atopic subjects than in the normal population. Contact urticaria of hands may lead to exacerbation of clinical disease in AD particularly in food handlers and slaughterhouse workers (food allergens) as well as health care workers (latex protein sensitivity) (Burns et al., 2004).

There has been dispute about whether atopic individuals are at greater risk to developing contact allergic reactions. CAD has a reported prevalence of as high as $28 \%$ in certain European countries, and similarity in clinical symptoms between AD and CAD makes correct diagnosis of CAD a challenge and can easily lead to misdiagnosis. A recent PT study found that a family history of $\mathrm{AD}(85 \%)$, female sex $(74 \%)$, and age $11-16(63 \%)$ were 
predisposing risk factors to contact allergen sensitization. Another study evaluating contact sensitization in a series of atopic pediatric patients, that were patch tested, identified $43 \%$ positive reactions without commenting on clinical relevance of these reactions (Fonacier \& Aquino, 2010 \& Jacob S et al., 2008). AD individuals can develop sensitivity to a variety of contact allergens such as topical medicaments including emollients and corticosteroids as well as fragrances, particularly in the presence of recalcitrant periorbital dermatitis. A positive past medical history of $\mathrm{AD}$ ( $44 \%$ in patients with periorbital dermatitis) has been found to be a predisposing factor for periorbital dermatitis when compared with all patchtested individuals (29\% with peri-orbital dermatitis). Statistically significantly more patients with $\mathrm{AD}$ were found in the group of patients with than without periorbital dermatoses. The periorbital region is a prime location for $\mathrm{AD}$ and in consequence a defective skin barrier alleviates sensitization to contact allergens as well as aeroallergens.

Hand eczema and compositae allergy may be more common in $\mathrm{AD}$, whereas allergies to topical corticosteroids and antiseptic solutions are only positive in a small subset of AD patients (15\%) (Fonacier \& Aquino, 2010).

Oat sensitization and its clinical manifestations in the form of digestive, respiratory and cutaneous symptoms (including CAD) has been identified in $15-20 \%$ of AD children, compared to none in the normal population. It is likely to be due to repeated applications of oat proteins through topical emollients and hygiene products on a predisposed impaired epidermal skin barrier of AD individuals. Oat can also be added to the list of food products (e.g. peanut, ovalbumin, almond) responsible for FA triggered by a possible percutaneous sensitization. Avoiding application of topical containing oat protein products in AD infants is paramount in reducing sensitization to oat (Boussault $P$ et al., 2007).

- New-onset dermatitis

- Progression or deterioration of existing dermatitis (AD/Psoriasis)

- Recalcitrant dermatitis by standard therapies

- Clinical presentation of dyshidrosis

Table 11. Factors that make a diagnosis of CAD likely

A lower frequency of positive quinoline allergy has been documented in AD individuals, whereas contact allergy to neomycin among atopic subjects is usually found to be equivalent or slightly raised in comparison to non-AD individuals. AD subjects appear to have increased efficiency in orally tolerising haptens but are inefficient in orally tolerising proteins. The reduced contact allergy to quinoline in AD patients might be explained by a higher exposure of haptens in the gut and skin at a young age producing "hapten"-tolerance (McFadden \& White, 2008).

History for CAD should always include the patient's personal hygiene environment, medical and medicament history as well as home and care giver environment. PT should be performed in all AD patients where CAD is considered and particularly in children with AD. However because of irritancy interpretation of PT may be difficult in AD individuals and the severity of AD might have an impact on the results obtained through PT. PT should always include the emollients used.

In summary more and more studies suggest, that CAD might occur as common in $\mathrm{AD}$ individuals and particularly in children with $\mathrm{AD}$ as in the general population. 
Consideration of CAD is recommended in each case of recurrent and recalcitrant clinical disease, and PT should be considered and offered to AD patients in those circumstances. However the relevance between a positive PT reaction and clinical severity of AD remains to be answered.

\section{Conclusion}

$\mathrm{AD}$ is a common chronic skin condition associated with high morbidity and major public health implications. As prevention of disease is not yet a real option, reducing morbidity is main aim of treatment. Identifying the underlying pathomechanism of the individual's AD is very crucial. High levels of specific IgE antibodies and or total $\operatorname{IgE}$ levels in serum are significantly associated with severity of dermatitis in individuals with the extrinsic type of $\mathrm{AD}$. Raised specific IgE antibodies can be detected in the peripheral blood for most trigger factors and allergens. Food and aeroallergens, microbes including S. aureus and Malassezia yeasts, contact allergens and autoallergens have been identified to trigger $\mathrm{AD}$ and perpetuate so the underlying immune and inflammatory cascade of AD.

To date no standard test is available to diagnose AD. Sensitization to various allergens is a major part of triggering and perpetuating the inflammatory skin response in AD. Various tests have been developed to investigate the underlying type(s) of hypersensitivity reaction involved in $\mathrm{AD}$ patients. None of the available tests so far have proven sensitive and specific enough to identify reliably relevance between clinical reaction and test result. Allergy tests commonly used in practice include measurement of total levels of $\mathrm{IgE}$ and allergen specific IgE levels in serum (RAST), SPT, APT, PT and DBPCFC.

Precise understanding of these tests including their limitations together with accurate correlation of patient history, symptoms and signs are required in order to differentiate between allergy, intolerances and hypersensitivities, and achieve an appropriate clinical diagnosis. Results must always be interpreted in the context of clinical history.

In a recent meta-analysis filaggrin gene defects have shown to increase the risk of developing allergic sensitization, AD, and allergic rhinitis. The presence of filaggrin gene mutations correlated strongly with disease severity and treatment failure, and also increased the risk of asthma in AD patients. Gene testing for filaggrin gene mutations might be an additional way to identify atopic individuals in future. Restoring skin barrier function in filaggrin deficient individuals early in life may help prevent the development of sensitization and halt the development and progression of allergic disease.

\section{References}

Boussault P et al. (2007). Oat sensitization in children with atopic dermatitis: prevalence risks and associated factors Allergy 2007: 62: 1251-1256

Burns T, Brethnach S, Cox N, Griffiths C (2004). Rook's Textbook of Dermatology, (7th ed. Chapter 18). Blackwell Publishing. ISBN 0-632-06429-3

Czarnobilska Ket al (2010). A half of school children with "ISAAC eczema" are ill with allergic contact dermatitis. J Eur Acad Dermatol Venereol; 4.

Darabi K et al. (2009). The role of Malassezia in atopic dermatitis affecting the head and neck of adults. J Am Acad Dermatol;60(1):125-36

Fonacier LS, Aquino MR (2010). The role of contact allergy in atopic dermatitis. Immunol Allergy Clin North Am;30(3):337-50 
Goodwin H (2008). Eczema and allergy: how useful is allergy testing? Paediatr Nurs; 20(10): 25-30

Jacob S et al.(2008) Patch testing: Another steroid-sparing agent to consider in children. Paed Dermatol;25 (1):81-87

Kedzierska A et al. (2008). Susceptibility testing and resistance phenotype detection in Staphylococcus aureus strains isolated from patients with atopic dermatitis, with apparent and recurrent skin colonization. Br J Dermatol;159(6):1290-9

Kim JS (2008). Paediatric atopic dermatitis: The importance of food allergens. Semin Cutan Med Surg;27(2):156-60

Lipozencić J, Wolf R (2010). The diagnostic value of atopy patch testing and skin prick testing in atopic dermatitis:facts and controversies. Clin Dermatol;28(1):38-44

Macias ES et al (2011) Superantigens in dermatology, J Am Acad Dermatol;64(3):455-72

McFadden JP, White JM (2008). Reduced frequency of atopic dermatitis quinoline-allergic patients: the "hapten-atopy hypothesis". Contact Dermatitis.;58(5):291-5

Most tables in the text have been taken from Reitamo S, Luger TA, Steinhoff M (2008). Textbook of Atopic Dermatitis, (Chapters 3-5 and 7-9) Informa healthcare, ISBN 978-1-84184-246-2

Reese I et al (2009). Diagnostic approach for suspected peudoallergic reaction to food ingredients. J Dtsch Dermatol Ges;7(1):70-7

Reitamo S, Luger TA, Steinhoff M (2008). Textbook of Atopic Dermatitis, (Chapters 3-5 and 7-9) Informa healthcare, ISBN 978-1-84184-246-2

Robinson M, Smart J (2008). Allergy testing in children, Aust Fam Physician.;37(4):210-3

Ronchetti $\mathrm{R}$ et al. (2008). Reproducibility of atopy patch tests with food and inhalant allergens. J Biol Regul Homeost Agents;22(1):27-33

Schlievert PM et al. (2008). Superantigen Profile of Staphylococcus aureus Isolates from Patients with Steroid-Resistant AtopicDermatitis. Clin Infect Dis;15;46(10):1562-7

Van den Oord RA, Sheikh A (2009). Filaggrin gene defects and risk of developing allergic sensitisation and allergic disorders: systematic review and meta-analysis. Brit Med $\mathrm{J} ; 339: \mathrm{b} 2433$ 


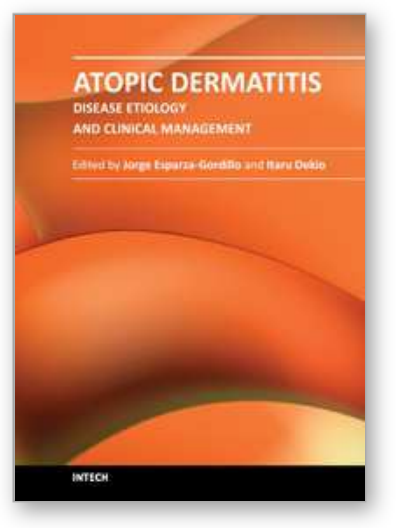

\author{
Atopic Dermatitis - Disease Etiology and Clinical Management \\ Edited by Dr. Jorge Esparza-Gordillo
}

ISBN 978-953-51-0110-9

Hard cover, 414 pages

Publisher InTech

Published online 22, February, 2012

Published in print edition February, 2012

Atopic Dermatitis is a common disease characterized by inflamed, itching and dry skin. This relapsing allergic disorder has complex etiology and shows a remarkably high clinical heterogeneity which complicates the diagnosis and clinical management. This book is divided into 4 sections. The first section (Disease Etiology) describes some of the physiological mechanisms underlying Atopic Dermatitis, including alterations in the immune system and the skin-barrier function. The important role of host-microorganism interactions on the pathophysiology of Atopic Dermatitis is discussed in the second section (Microorganisms in Atopic Dermatitis). An overview of the clinical diagnostic criteria and the disease management protocols commonly used is given in the third section (Diagnosis and Clinical Management). The last section (New Treatments) describes new therapeutic approaches that are not widely used but are currently being studied due to preliminary evidence showing a clinical benefit for Atopic Dermatitis.

\title{
How to reference
}

In order to correctly reference this scholarly work, feel free to copy and paste the following:

Evmorfia Ladoyanni (2012). Trigger Factors, Allergens and Allergy Testing in Atopic Dermatitis, Atopic Dermatitis - Disease Etiology and Clinical Management, Dr. Jorge Esparza-Gordillo (Ed.), ISBN: 978-953-510110-9, InTech, Available from: http://www.intechopen.com/books/atopic-dermatitis-disease-etiology-andclinical-management/trigger-factors-allergens-and-allergy-testing-in-atopic-dermatitis

\section{INTECH}

open science | open minds

\section{InTech Europe}

University Campus STeP Ri

Slavka Krautzeka 83/A

51000 Rijeka, Croatia

Phone: +385 (51) 770447

Fax: +385 (51) 686166

www.intechopen.com

\section{InTech China}

Unit 405, Office Block, Hotel Equatorial Shanghai

No.65, Yan An Road (West), Shanghai, 200040, China

中国上海市延安西路65号上海国际贵都大饭店办公楼 405 单元

Phone: +86-21-62489820

Fax: $+86-21-62489821$ 
(C) 2012 The Author(s). Licensee IntechOpen. This is an open access article distributed under the terms of the Creative Commons Attribution 3.0 License, which permits unrestricted use, distribution, and reproduction in any medium, provided the original work is properly cited. 